\title{
Specific accumulation of cholesterol-rich liposomes in the inflammatory tissue of rats with adjuvant arthritis
}

\author{
W G Love, N Amos, I W Kellaway, B D Williams
}

\begin{abstract}
High performance liquid chromatography has shown that after intravenous injection cholesterol-poor liposomes (100 nm) are unstable and their phospholipid is redistributed. Under identical conditions cholesterol-rich liposomes remain structurally intact within the circulation. When injected intravenously cholesterol-rich liposomes accumulate within the inflamed paws of rats with adjuvant induced arthritis to the same extent as cholesterol-poor liposomes. Uptake in inflamed tissue of three cholesterol-rich liposome preparations was always significantly greater than the uptake noted in normal tissue. The degree of accumulation in inflamed tissue was found to depend on the size of the liposome, with the greatest uptake, $7 \%$ of the injected dose, achieved by the smallest vesicle $(100 \mathrm{~nm})$. These results indicate that intact liposomes accumulate at inflamed joint tissue sites. Therefore the passive targeting of antiinflammatory drugs encapsulated within these liposomes could be contemplated.
\end{abstract}

We have previously shown that liposomes (microscopic phospholipid vesicles), which carry a net negative surface charge, given intravenously specifically accumulate in the inflamed paws of rats with adjuvant induced arthritis. ${ }^{1}$ Liposomes of identical composition when isotopically labelled have also been shown scintigraphically to image the actively inflamed joints of patients with rheumatoid arthritis. ${ }^{23}$ The aim of these studies was to elucidate a liposome formulation with a high affinity for inflammatory tissue as well as establishing the underlying mechanism(s) responsible for the specific liposome accumulation found in the rat paws and the scintigraphic scans in rheumateid arthritis. Previous liposome stability studies showed that cholesterol-poor liposomes were relatively unstable in serum, and a redistribution of the liposomal phospholipid was shown to occur. ${ }^{4}$

It is known that when a high ratio of cholesterol is incorporated into liposomes-for example, equimolar with the other phospholipids, condensed molecular packing occurs within the bilayers, which results in a more stable liposome structure. ${ }^{5} 6$ We have confirmed this finding and shown that negatively charged, cholesterol-rich liposomes are stable both in serum in vitro and in vivo. ${ }^{4}$ Investigations have now been undertaken to establish if cholesterol-rich liposomes accumulate within inflammatory tissue to the same extent as cholesterol-poor liposomes, and to examine the effect of liposome size on tissue uptake.

\section{Methods and materials}

Egg phosphatidylcholine and phosphatidic acid were purchased from Lipid Products (UK), and cholesterol from BDH (UK). Sodium chromate $\left({ }^{51} \mathrm{Cr}\right)$ was obtained from Amersham (UK) as was ${ }^{99} \mathrm{TcO}_{4}^{-}$eluted from a sterile ${ }^{99 \mathrm{~m}} \mathrm{Tc}$ generator.

Sprague-Dawley rats were obtained from Tuck and Sons (UK), Mycobacterium butyricum from Difco Laboratories (Detroit), and heavy paraffin oil from Sigma (UK).

\section{ADJUVANT INDUCED ARTHRITIC RAT MODEL ${ }^{7}$}

Female Sprague-Dawley rats weighing $200 \mathrm{~g}$ were injected intradermally with $100 \mu \mathrm{l}$ of heavy paraffin oil containing $1 \mathrm{mg}$ of finely ground heat killed Mycobacterium butyricum. Arthritis developed 10-14 days after injection and the severity of inflammation was given an arthritic score. ${ }^{8}$ The arthritic score for each paw was graded from 0 to 5,0 representing no inflammation, the paw being indistinguishable from the control, and 5 representing a paw which was severely oedematous and erythematous. All rats used in this study had an arthritic score greater than 3 for both hind and fore paws.

\section{LIPOSOME PREPARATION}

Negatively charged, cholesterol-poor liposomes were prepared in a molar ratio of 7:2:1 of egg phosphatidylcholine:cholesterol:phosphatidic acid, and cholesterol-rich liposomes in a molar ratio of 7:7:1. The total lipid mass used was 40 $\mathrm{mg}$. The original method of Bangham et $a l^{9}$ was modified as described below.

The lipids were dissolved in chloroform and dried onto a glass vessel wall under a stream of nitrogen gas. Multilamellar vesicles (large liposomes $>1 \mu \mathrm{m}$ ) were formed by addition of $2 \mathrm{ml}$ of $0.9 \%$ saline to the lipid film and then shaking the mixture. The liposomes were reduced in size by probe sonication with an MSE $150 \mathrm{~mW}$ Soniprep with a $10 \mathrm{~mm}$ probe. Liposome size was regulated by the length of the sonication time. Titanium fragments from the probe were removed from the liposome preparations by centrifugation for 20 minutes at $10000 \mathrm{~g}$. The sizes of the choelsterol-rich liposome preparations used in this study were $100 \mathrm{~nm}, 140 \mathrm{~nm}$, and $200 \mathrm{~nm}$, as determined by photon correlation spectroscopy (Malvern, England). 
LIPOSOME RADIOLABELLING TECHNIQUE

Preformed liposomes were radiolabelled with ${ }^{99} \mathrm{~m}$ Tc using the method described by Richardson et $a l^{10}$ and modifications to this method as described by Love et al. ${ }^{11}$

TISSUE DISTRIBUTION OF ${ }^{99 \mathrm{~m}}$ Tc LABELLED, NEGATIVELY CHARGED LIPOSOMES

Control and arthritic rats (three rats in each group) were used for each of the different sized liposome preparations $(25 \mathrm{mg} / \mathrm{kg}, 4 \mathrm{MBq})$. A small aliquot of each was retained to serve as a standard. The liposomes were injected intravenously into the tail vein. At the same time each animal received $100 \mu \mathrm{l}$ of a $50 \%$ suspension of ${ }^{51} \mathrm{Cr}(370 \mathrm{kBq})$ labelled rat erythrocytes to serve as a blood pool marker. ${ }^{12}$

All the rats were bled at regular intervals from the tail vein during the experiment so that the clearance of each of the preparations could be determined. After $80 \%$ of each preparation had been cleared from the circulation the rats were killed and the relevant tissues, including all four paws, were removed. The amounts of ${ }^{99} \mathrm{~m} \mathrm{Tc}$ and ${ }^{51} \mathrm{Cr}$ present in the tissues were measured in an LKB Compugamma counter, with the appropriate corrections made for cross over between the two channels. Specific liposome uptake in various tissues was calculated with the following equation:

Specific liposome uptake $={ }^{99 \mathrm{~m}} \mathrm{Tc}$ tissue $-{ }^{51} \mathrm{Cr}$ tissue $\times \frac{{ }^{99 \mathrm{~m}} \mathrm{Tc} \text { blood }}{{ }^{51} \mathrm{Cr} \text { blood }}$

ANALYSIS OF LIPOSOME STABILITY

The protocol of Love $e t a l^{4}$ was used for the high performance liquid chromatography (HPLC) of the liposomes. Thus ${ }^{99 \mathrm{~m}} \mathrm{~T}$ c labelled cholesterolpoor and cholesterol-rich liposome preparations $(100 \mathrm{~nm})$ were injected intravenously into normal Sprague-Dawley rats. Plasma obtained

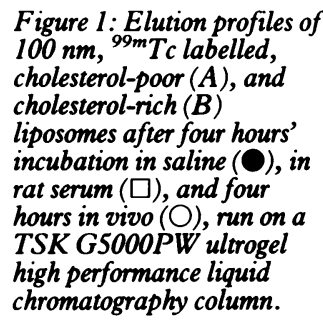

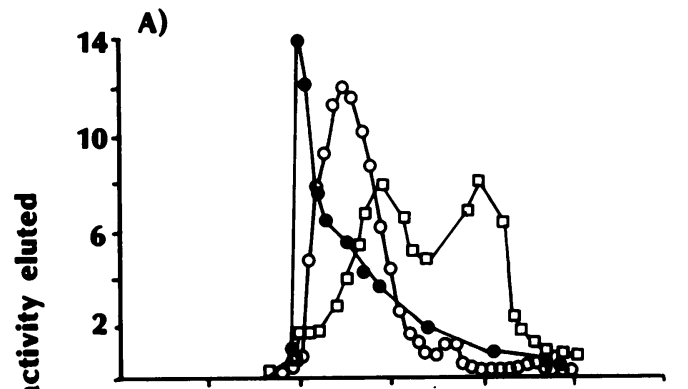

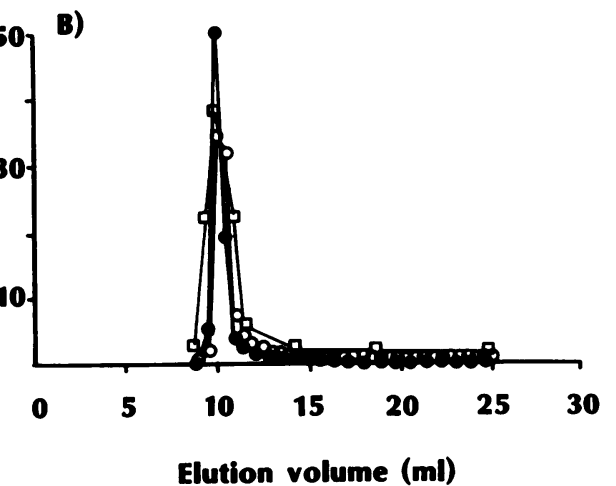

from blood samples taken four hours after injection was run on a TSK G5000PW HPLC column (fractionation range $7 \times 10^{6}-5 \times 10^{5}$ daltons). Fractions were collected and counted for ${ }^{99 m} \mathrm{Tc}$ activity and the profiles obtained in vivo compared with the HPLC profiles of the liposomes which had been incubated separately at $37^{\circ} \mathrm{C}$ at a $1: 100$ dilution in rat serum and in saline.

\section{Results}

Figure 1 shows the HPLC elution profiles of the cholesterol-poor and cholesterol-rich $100 \mathrm{~nm}$ liposomes four hours after intravenous injection into normal rats and after incubation in rat serum and in saline. In vivo and in rat serum the cholesterolpoor liposomes were reduced in size and radioactivity peaks were seen to enter the gel (fig 1A), whereas the cholesterol-rich liposomes were not reduced in size (fig $1 B$ ).

Figure 2 shows the clearance of the cholesterolrich liposomes from the rat circulation. The time taken for an $80 \%$ clearance of each liposome preparation was 180 minutes $(200 \mathrm{~nm}), 240$ minutes $(140 \mathrm{~nm})$, and 300 minutes $(100 \mathrm{~nm})$. Included in fig 2 are the respective clearance rates for $100 \mathrm{~nm}$ and $200 \mathrm{~nm}$ cholesterol-poor liposomes.

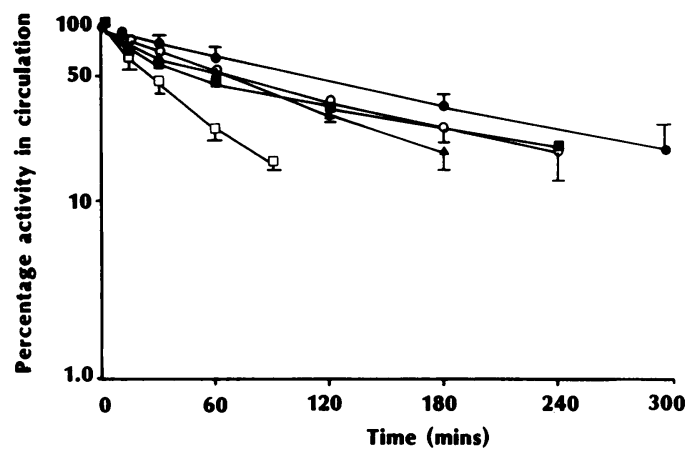

Figure 2: Clearance profiles of various sized cholesterol-rich and cholesterol-poor liposomes from the circulation of rats (cholesterol-rich-200 $\mathrm{nm}(\mathbf{\Delta}), 140 \mathrm{~nm}(\mathrm{\square}), 100 \mathrm{~nm}(\mathrm{O})$; cholesterol poor-200 $\mathrm{nm}(\square), 100 \mathrm{~nm},(\mathrm{O}))$. Mean (SD) of three rats in each group.

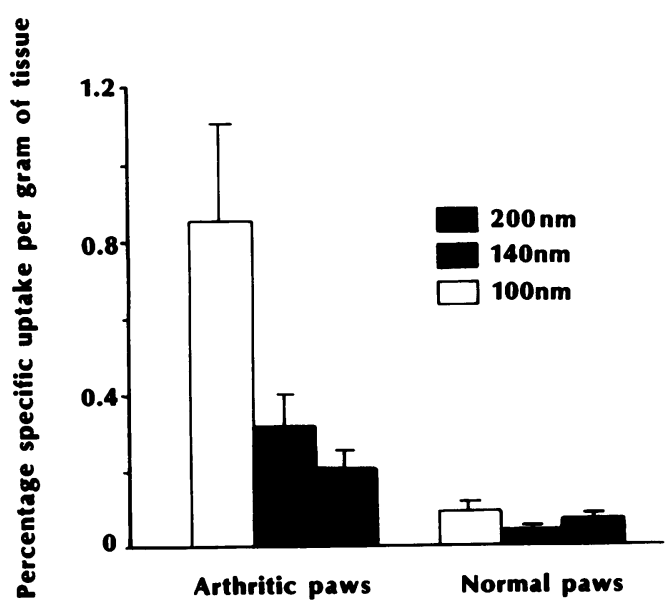

Figure 3: Specific uptake of cholesterol-rich liposomes in paws of rats with adjuvant induced arthritis and normal rats. Mean (SD) of three rats in each group. Student's test shows significant differences between uptake of different sized liposomes in arthritic and normal paws $(p<0.001)$ except for $200 \mathrm{~nm} v 140 \mathrm{~nm}$ in arthritic paws where $p<0.05$ $(n=12-24)$. 
Figure 4: Comparison of the specific uptake of $100 \mathrm{~nm}$ and $200 \mathrm{~nm}$ cholesterol-rich and cholesterol-poor liposomes by normal and arthritic rat paws. Mean $(S D)$ of three rats in each group. Statistical analysis of the results is shown in table 1 .

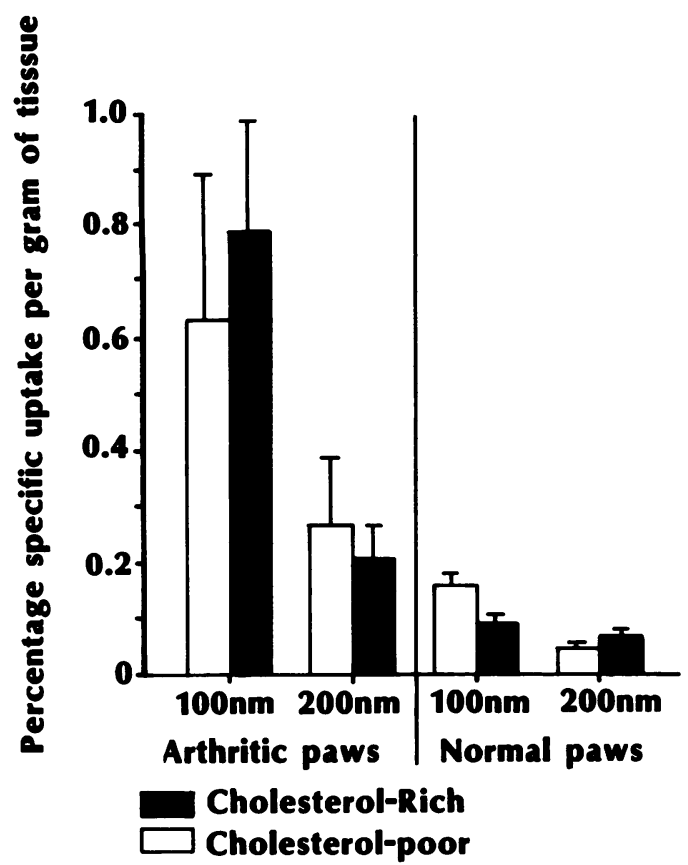

Accumulation of each of the three liposome preparations in arthritic paw tissue was significantly greater than in normal paw tissue $(p<0.001)$. The degree of uptake of the cholesterol-rich liposomes by arthritic paws was proportional to the liposome diameter (fig 3), with greatest accumulation achieved by the smallest liposomes ( $0.85 \%$ per gram of $100 \mathrm{~nm}$ liposomes compared with $0 \cdot 20 \%$ per gram for the $200 \mathrm{~nm}$ liposomes). Although there were significant differences in the amount of uptake between the three cholesterolrich liposome preparations within normal paws, these did not bear any relation to the liposome size.

Some interesting points became apparent when the uptake of cholesterol-rich liposome by tissue

Table 1: Student's t test of significance for uptake of $100 \mathrm{~nm}$ and $200 \mathrm{~nm}$ cholesterol-rich (CR) and cholesterol-poor (CP) liposomes by normal and arthritic rat paws (see fig 4)

\begin{tabular}{|c|c|c|}
\hline Comparison & Arthritis paws & Normal paws \\
\hline $100 \mathrm{~nm} v 100 \mathrm{~nm}$ & $p>0.1$ & $p<0.001$ \\
\hline$\underset{\mathrm{CR}}{200 \mathrm{~nm}} \underset{\mathrm{CP}}{200} \mathrm{~nm}$ & $p>0.1$ & $\mathrm{p}<0.001$ \\
\hline $\begin{array}{l}100 \mathrm{~nm} v 200 \mathrm{~nm} \\
\mathrm{CR}, \mathrm{CP} \text { CR, CP* } \\
{ }^{*} \text { Except }\end{array}$ & $\mathrm{p}<0.001$ & $p<0.001$ \\
\hline $\begin{array}{ccc}100 \mathrm{~nm} & v 200 \mathrm{~nm} \\
C R & C P\end{array}$ & $\mathrm{p}<0.01$ & $\mathrm{p}<0.001$ \\
\hline
\end{tabular}

Arthritic paws $v$ normal paws (all sizes and compositions) $\mathrm{p}<0 \cdot 001, \mathrm{n}=12-24$. was compared with the distribution of cholesterolpoor liposomes. Figure 4 illustrates the relative accumulation of $100 \mathrm{~nm}$ and $200 \mathrm{~nm}$, cholesterolrich and cholesterol-poor liposomes in arthritic and normal rat paw tissue and table 1 provides a statistical analysis of the results. Cholesterol-poor and rich liposomes were equally sequestrated in inflammatory paw tissue for both $100 \mathrm{~nm}$ and 200 $\mathrm{nm}$ vesicles $(\mathrm{p}>0 \cdot 1)$. Small but significant differences were observed for normal paw tissue uptake of the cholesterol-poor and cholesterol-rich 100 $\mathrm{nm}$ and $200 \mathrm{~nm}$ liposomes, however. Table 2 contains the tissue distribution data (expressed as a percentage of the total liposome dose injected) for the cholesterol-poor and cholesterol-rich $100 \mathrm{~nm}$ and $200 \mathrm{~nm}$ liposomes. Most of each liposome preparation accumulated in the liver and spleen. Cholesterol-rich liposomes of both sizes accumulated in the liver, heart, lungs, and arthritic paws to the same extent as cholesterolpoor liposomes, but considerably greater splenic uptake of cholesterol-rich liposomes was found $(p<0.001)$. The only other differences in distribution were for the $100 \mathrm{~nm}$ liposome preparations, for which significantly greater amounts of cholesterol-poor liposomes accumulated in the kidneys and normal paw tissue.

\section{Discussion}

Our initial finding from this study was the increased circulation time of the cholesterol-rich liposomes compared with that of the cholesterolpoor vesicles of the same size. This phenomenon has been shown by others. ${ }^{13} 14$ As cholesterol increases the liposomal bilayer rigidity it is thought to cause a decrease in liposome interaction with both humoral and cellular components, such as serum proteins, other opsonins, and phagocytic cells, all of which act to prolong the circulation time of the cholesterol-rich liposomes. The rate of clearance from the circulation of the three cholesterol-rich liposome preparations tested was directly related to liposome size, with the largest being cleared most rapidly.

The specific accumulation of cholesterol-rich liposomes in inflamed tissue was the most striking feature of this study. The degree of uptake was directly related to liposome size, with greatest accumulation of the smallest liposomes. When these results were compared with those obtained for cholesterol-poor liposomes of the same $\operatorname{size}^{1}(100 \mathrm{~nm}$ and $200 \mathrm{~nm})$ there was no significant difference in the amount of uptake of either liposome preparation by arthritic paws $(p>0 \cdot 1)$.

Table 2: Total specific accumulation* of the cholesterol-poor and cholesterol-rich $100 \mathrm{~nm}$ and $200 \mathrm{~nm}$ liposome preparations in rat tissues after $80 \%$ clearance from the circulation. Values are means $(S D)$ with six rats in each group

\begin{tabular}{|c|c|c|c|c|c|c|c|}
\hline & Liver & Spleen & Heart & Kidneys & Lungs & $\begin{array}{l}\text { Arthritic } \\
\text { paws }\end{array}$ & $\begin{array}{l}\text { Normal } \\
\text { paws }\end{array}$ \\
\hline $\begin{array}{l}100 \text { nm liposomes } \\
\text { Cholesterol-poor } \\
\text { Cholesterol-rich } \\
\text { Student's } t \text { test }\end{array}$ & $\begin{array}{l}32(6.9) \\
36(9 \cdot 6) \\
p>0.1\end{array}$ & $\begin{array}{l}6.1(1.2) \\
17.3(3.8) \\
p<0.001\end{array}$ & $\begin{array}{l}0.09(0.01) \\
0.10(0.03) \\
p>0.1\end{array}$ & $\begin{array}{l}6.4(0.5) \\
2.5(0.3) \\
p<0.001\end{array}$ & $\begin{array}{l}0.47(0.07) \\
0.38(0.13) \\
p>0.1\end{array}$ & $\begin{array}{l}6.4(2.8) \\
7.0(2.5) \\
p>0.5\end{array}$ & $\begin{array}{l}0.7(0.28) \\
0.42(0.25) \\
p<0.01\end{array}$ \\
\hline $\begin{array}{l}200 \mathrm{~nm} \text { liposomes } \\
\text { Cholesterol-poor } \\
\text { Cholesterol-rich } \\
\text { Student's } t \text { test }\end{array}$ & $\begin{array}{l}42(14) \\
44(7.9) \\
p>0.5\end{array}$ & $\begin{array}{l}5.1(2.2) \\
12.9(2.8) \\
p<0.001\end{array}$ & $\begin{array}{l}0.09(0.02) \\
0.09(0.04) \\
p>0.5\end{array}$ & $\begin{array}{l}2.4(0.5) \\
3.1(0.6) \\
p>0.02\end{array}$ & $\begin{array}{l}0.38(0.41) \\
0.20(0.12) \\
p>0.1\end{array}$ & $\begin{array}{l}2.4(0.9) \\
1.7(0.3) \\
p>0.5\end{array}$ & $\begin{array}{l}0.23(0.11) \\
0.32(0.13) \\
p>0.05\end{array}$ \\
\hline
\end{tabular}

${ }^{*}$ Expressed as a percentage of the total liposome dose injected. 
The enhanced stability achieved by incorporation of a high concentration of cholesterol into liposomes is also well recognised, ${ }^{4-6}$ and the integrity of the liposome structure in vivo was shown by HPLC. The uptake of the cholesterolrich liposomes by inflamed paws was an important observation because it indicated that fully intact liposomes can gain access to inflamed tissue sites.

There were two major differences between the fate of cholesterol-rich and cholesterol-poor liposomes in vivo. The first was the relative stability (as assessed by HPLC) of the cholesterol-rich liposomes, whose structure remained unchanged, whereas in cholesterol-poor vesicles phospholipids were redistributed to lower molecular mass moieties. These moieties were found when incubation took place in serum, but they were not present in the profile obtained in vivo, presumably owing to removal from the rat circulation.

The second difference was in the uptake of liposomes by the spleen. It was found that $17.3 \%$ and $12.9 \%$ of the total dose injected of $100 \mathrm{~nm}$ and $200 \mathrm{~nm}$ cholesterol-rich liposomes accumulated in the spleen compared with $6 \cdot 1 \%$ and $5 \cdot 1 \%$ for the $100 \mathrm{~nm}$ and $200 \mathrm{~nm}$ cholesterol-poor liposomes. Roerdink et al and Moghimi and Patel found a similar distribution in the spleen and a decrease in the uptake by the liver, and suggested that macrophages within these two organs may require different opsonising factors. ${ }^{15}$ Their observed decrease in liver uptake was due to the fact that the animal groups receiving cholesterol-rich and cholesterol-free liposomes were killed at equivalent times, whereas this study compares liposome distribution after $80 \%$ clearance of each liposome preparation.

Over $6 \%$ of the $100 \mathrm{~nm}$ cholesterol-poor liposomes accumulated in the kidneys-an amount significantly greater $(\mathrm{p}<0.001)$ than the $2.5 \%$ uptake seen with the $100 \mathrm{~nm}$ cholesterolrich liposomes. This difference in renal uptake was not found with the $200 \mathrm{~nm}$ liposomes, so may be attributable to a reduction in the size of the $100 \mathrm{~nm}$ liposomes. A similar result was not seen with the $200 \mathrm{~nm}$ cholesterol-poor liposomes because of increased stability increasing liposome size. ${ }^{17} 18$ This finding coupled with the low molecular mass moieties found in vitro may explain the preferential accumulation of the 100 nm cholesterol-poor liposomes in normal paw tissue and the kidneys. The major observation drawn from this study is that cholesterol-rich liposomes accumulate in inflammatory tissue. The cholesterol-rich liposomes have been shown to remain structurally intact in vivo, and when injected intravenously up to $7 \%$ of the $100 \mathrm{~nm}$ vesicles specifically accumulated within the inflamed rat paws. The relevance of these findings in the rat to the scintigraphic studies carried out in patients with active rheumatoid arthritis remains to be established. If as previously postulated, however, phagocytic cells are a major cause of liposome sequestration within inflamed tissue ${ }^{1}$ then a decreased uptake of cholesterol-rich liposomes would have been expected because of the inability of cells to fuse or phagocytose these stable liposomes. ${ }^{1920}$ Inflamed tissue phagocytes may have selective affinity for different liposome formulations as shown by the relative distribution of cholesterolrich and cholesterol-poor liposomes within the liver and spleen. ${ }^{1516}$ Further studies are being undertaken to clarify this issue.

The results shown here indicate that stable liposomes have a potential for use as a carrier system for a variety of drugs, and would probably have greatest benefit for the passive targeting of the more toxic antirheumatic agents.

We are grateful to the Arthritis and Rheumatism Council for their financial support.

1 Love W G, Amos N, Williams B D, Kellaway I W. Specific accumulation of technetium $-99 \mathrm{~m}$ radiolabelled, negative liposomes in the inflamed paws of rats with adjuvant induced arthritis: effect of liposome size. Ann Rheum Dis 1989; 48: 143-8.

2 Williams B D, O'Sullivan M M, Saggu G S, Williams K E, Williams L A, Morgan J R. Synovial accumulation of
technetium labelled liposomes in rheumatoid arthritis. Ann technetium labelled liposomes

3 O'Sullivan M M, Powell N, French A P, Morgan J R, Williams B D. Inflammatory joint disease: a comparison of liposome scanning, bone scanning, and radiography. Ann Rheum Dis 1988; 47: 485-91.

4 Love W G, Amos N, Williams B D, Kellaway I W. High performance liquid chromatographic analysis of liposome stability. Fournal of Microencapsulation 1990; 7: 105-12.

5 Kirby C, Clarke J, Gregoriadis G. Effect of cholesterol content of small unilamellar liposomes on their stability in vivo and in vitro. Biochem $f$ 1980; 186: 591-8.

6 Senior J, Gregoriadis G. Stability of small unilamellar liposomes in serum and clearance from the circulation: liposomes in serum and clearance from the circulation:
effect of the phospholipid and cholesterol components. Life Sci 1982; 30: 2123-36.

7 Pearson C M. Development of arthritis, periarthritis and periostitis in rats given adjuvants. Proc Soc Exp Biol Med 1956; 91: 95-101.

8 Trentham D E, Townes A S, Kang A H. Autoimmunity to type II collagen: an experimental model of arthritis. $\mathcal{F}$ Exp Med 1977; 146: 857-68.

9 Bangham A D, Standish M M, Watkins J C. The action of steroids and streptolysin $S$ on the permeability of phospholipid structures to cations. F Mol Biol 1965; 13: 238-52.

10 Richardson V R, Jeyasingh K, Jewkes R F, Ryman B E Tattersall M H. Properties of ${ }^{99 \mathrm{~m}} \mathrm{Tc}$-labelled liposomes in normal, and tumour-bearing rats. Biochem Soc Trans 1977; 5: $290-2$.

11 Love W G, Amos N, Williams B D, Kellaway I W. Effect of liposome surface charge on the stability of $99 \mathrm{~m}$ Technetium radiolabelled liposomes. Fournal of Microencapsulation 1989; 6: 105-13.

12 Gray S J, Sterling K. The tagging of red cells and plasma proteins with radioactive chromium. $\mathcal{F}$ Clin Invest 1950; 29 . 1604-13.

13 Patel H M, Tuzel N S, Ryman B E. Inhibitor effect of cholesterol on the uptake of liposomes by liver and spleen. Biochem Biophys Acta 1983; 761: 142-51.

14 Dave J, Patel H M. Differentiation in hepatic and splenic phagocytic activity during reticuloendothelial blockade with cholesterol-free and rich liposomes. Biochim Biophys with cholesterol-free and

15 Roerdink F H, Regts J, Handel T, Sullivan S M, Baldeschwieler J D, Scherphof G L. Effect of cholesterol on the uptake and intracellular degradation of liposomes by live and spleen; a combined biochemical and gamma-ray perturbed angular correlation study. Biochim Biophys Acto 1989; 980: $234-40$

16 Moghimi S M, Patel H M. Tissue specific opsonins for phagocytic cells and their different affinity for cholesterolrich liposomes. FEBS Lett 1988; 233: 143-7.

17 Wetterau J R, Jonas A. Effect of DPPC vesicle curvature on the reaction with human apolipoprotein A-I. $\mathcal{F}$ Biol Chem 1982; 257: $10961-6$.

18 Scherphof G, Mnziseit H. On the size-dependent disintegration of small vuilamellar phosphatidylcholine vesicles in rat plasma. Fvidence of complete loss of vesicle structure. piachem 3 1984; 221: 423-9.

19 Papahadjopulos D, Poste G, Schaeffer B E. Fusion of mammalian cells by unilamellar lipid vesicles; influence of lipid surface charge, fluidity and cholesterol. Biochim Biophys Acta 1973; 323: 23-42.

20 Johnson S M. The inability of macrophages to digest liposomes containing a high proportion of cholesterol. Biochem Soc Trans 1975; 3: 160-1. 